\section{KEYNOTE-407 bestätigt Zusatznutzen von Pembrolizumab beim Plattenepithelkarzinom}

Paz-Ares L et al. Pembrolizumab plus Chemotherapy for Squamous Non-Small-Cell Lung Cancer. N Engl J Med 2018; doi:10.1056/ NEJMoa1810865

Die Standardtherapie des unbehandelten metastasierten Plattenepithelkarzinoms der Lunge besteht in einer platinbasierten Chemotherapie oder Pembrolizumab. Die Immuntherapie erfolgt in Abhängigkeit vom PD-1-Expressionsgrad. Die zweite Interimsanalyse der doppelblinden KEYNOTE-407-Studie belegte einen expressionsunabhängigen Überlebensvorteil für die Kombination von Chemo- und Immuntherapie.

Voraussetzungen für die Studienteilnahme waren u.a. ein nicht kleinzelliges Bronchialkarzinom mit Plattenepithelhistologie (sNSCLC), ein guter Allgemeinzustand, keine systemische Vorbehandlung und ein bekannter PD-1-Expressionsgrad. Pembrolizumab inhibiert den Checkpoint Programmed-Death-1 (PD-1), überwindet damit natürliche Bremsen der Immunantwort und steigert konsekutiv die körpereigene Tumorabwehr. Von 559 Patienten wiesen 63,1\% eine PD-1-Expression $>1 \%$ auf und galten damit als positiv.

In Keynote-407 erhielten die Erkrankten 4 Kurse Chemotherapie (Carboplatin plus Paclitaxel/Nanopartikel-Paclitaxel). In der Verumgruppe bekamen 278 Patienten jeweils an Tag 1 zusätzlich Pembrolizumab. Die Immuntherapie wurde nach Abschluss der Chemotherapie fortgeführt (max. 35 Kurse). 280 Erkrankte erhielten additiv zum Standard Plazebo.
Die durchschnittliche Behandlungszeit betrug 6,3 Monate (Verumgruppe) vs. 4,7 Monate (Plazebogruppe). 78,8\% und 73,2\% erhielten 4 Kurse Chemotherapie. Die Beobachtungszeit lag im Mittel bei 7,8 Monaten. 205 Patienten starben. Verglichen mit Chemotherapie/Plazebo resultierte Chemotherapie/Pembrolizumab in einem signifikant längeren Überleben:

- OS 15,9 vs. 11,3 Monate,

- Mortalität HR 0,64

(95\%-KI 0,49-0,85; $\mathrm{p}<0,001)$,

- 1 -Jahres-Überleben $65,2 \%$ vs. $48,3 \%$.

Dies galt für alle präspezifizierten Subgruppen. Insbesondere beeinflusste der PD-1-Expressionsgrad die Ergebnisse nicht signifikant. Der kombinierte Endpunkt Tod/Progress trat bei 349 Patienten ein. Pembrolizumab verlängerte die progressionsfreie Zeit und reduzierte die Wahrscheinlichkeit für Tod/Progress:

- PFS 6,4 vs. 4,8 Monate,

- Tod/Progress HR 0,56 (95\%-KI 0,45$0,7 ; \mathrm{p}<0,001)$.

Der Zusatznutzen stieg mit der PD-1-Expression. Bei einem Grad < $1 \%$ betrug das PFS 6,3 vs. 5,3 Monate, bei einer Expression von $1-49 \%$ ergaben sich 7,2 vs. 5,2 Monate und bei einem starken Expressionsgrad $\geq 50 \%$ lebten die Patienten 8 vs. 4,2 Monate progressionsfrei. 57,9\% (additiv Pembrolizumab) und 38,4\% (additiv Plazebo) sprachen nach durchschnittlich 1,4 Monaten auf die Behandlung an (RECIST-Kriterien). Die Response hielt im Mittel 7,7 Monate an.

Fast alle Patienten hatten Nebenwirkungen. Komplikationen $\geq$ Grad 3 betrafen $69,8 \%$ der Patienten mit der Immuntherapie und 68,2\% mit Plazebo. Ein behandlungsassoziierter Therapieabbruch kam bei $13,3 \%$ und 6,4\% vor. Am häufigsten waren Anämie, Neutropenie und Alopezie. Immunmediierte und Infu- sionsreaktionen traten in der Verumgruppe öfter auf (28,8\% vs. 8,6\%; Grad 3: $10,8 \%$ vs. $3,2 \%)$. Immunologisch vermittelte Nebenwirkungen waren Pneumonitiden und Autoimmunhepatitiden. In jeder Gruppe starb 1 Patient an einer Pneumonitis.

FAZIT

Pembrolizumab reduzierte die Mortalitätswahrscheinlichkeit um $36 \%$ und die Kombination Tod/Progress um $44 \%$. Neue Sicherheitsbedenken durch die Checkpoint-Inhibition ergaben sich nicht. Die höhere Abbruchrate im Verumarm bringen die Autoren mit der längeren Behandlungsdauer in Zusammenhang. PazAres et al. erwarten eine Dauerhaftigkeit des Zusatznutzens von Pembrolizumab, u. U. sogar eine Zunahme im längerfristigen Verlauf. Die kurze Beobachtungszeit schlösse aber auch langfristige toxische Effekte nicht aus.

Dr. med. Susanne Krome, Melle 